\title{
A Population-Based Model to Consider the Effect of Seasonal Variation on Serum 25(OH)D and Vitamin D Status
}

\author{
Philippe Vuistiner, ${ }^{1}$ Valentin Rousson, ${ }^{1}$ Hugues Henry, ${ }^{2}$ \\ Pierre Lescuyer, ${ }^{3}$ Olivier Boulat, ${ }^{2}$ Jean-Michel Gaspoz, ${ }^{4}$ Vincent Mooser, ${ }^{2}$ \\ Peter Vollenweider, ${ }^{5}$ Gerard Waeber, ${ }^{5}$ Jacques Cornuz, ${ }^{6}$ Fred Paccaud, ${ }^{1}$ \\ Murielle Bochud, ${ }^{1}$ and Idris Guessous ${ }^{1,4,7}$ \\ ${ }^{1}$ Institute of Social and Preventive Medicine (IUMSP), University Hospital of Lausanne (CHUV), 1010 Lausanne, Switzerland \\ ${ }^{2}$ Biomedicine, University Hospital of Lausanne, 1011 Lausanne, Switzerland \\ ${ }^{3}$ Department of Genetic and Laboratory Medicine, Geneva University Hospitals, 1205 Geneva, Switzerland \\ ${ }^{4}$ Unit of Population Epidemiology, Division of Primary Care Medicine, Department of Community Medicine, \\ Primary Care and Emergency Medicine, Geneva University Hospitals, 1205 Geneva, Switzerland \\ ${ }^{5}$ Department of Internal Medicine, Internal Medicine, University Hospital of Lausanne (CHUV), 1011 Lausanne, Switzerland \\ ${ }^{6}$ Department for Ambulatory Care and Community Medicine, University of Lausanne, 1011 Lausanne, Switzerland \\ ${ }^{7}$ Department of Epidemiology, Rollins School of Public Health, Emory University, Atlanta, GA 30322, USA
}

Correspondence should be addressed to Murielle Bochud; murielle.bochud@chuv.ch and Idris Guessous; idris.guessous@hcuge.ch

Received 30 April 2015; Accepted 16 August 2015

Academic Editor: Sebastian Straube

Copyright (c) 2015 Philippe Vuistiner et al. This is an open access article distributed under the Creative Commons Attribution License, which permits unrestricted use, distribution, and reproduction in any medium, provided the original work is properly cited.

Background. We elaborated a model that predicts the centiles of the $25(\mathrm{OH}) \mathrm{D}$ distribution taking into account seasonal variation. Methods. Data from two Swiss population-based studies were used to generate (CoLaus) and validate (Bus Santé) the model. Serum 25(OH)D was measured by ultra high pressure LC-MS/MS and immunoassay. Linear regression models on square-root transformed $25(\mathrm{OH}) \mathrm{D}$ values were used to predict centiles of the $25(\mathrm{OH}) \mathrm{D}$ distribution. Distribution functions of the observations from the replication set predicted with the model were inspected to assess replication. Results. Overall, 4,912 and 2,537 Caucasians were included in original and replication sets, respectively. Mean (SD) $25(\mathrm{OH}) \mathrm{D}$, age, BMI, and \% of men were $47.5(22.1) \mathrm{nmol} / \mathrm{L}$, $49.8(8.5)$ years, $25.6(4.1) \mathrm{kg} / \mathrm{m}^{2}$, and $49.3 \%$ in the original study. The best model included gender, BMI, and sin-cos functions of measurement day. Sex- and BMI-specific $25(\mathrm{OH}) \mathrm{D}$ centile curves as a function of measurement date were generated. The model estimates any centile of the $25(\mathrm{OH}) \mathrm{D}$ distribution for given values of sex, BMI, and date and the quantile corresponding to a $25(\mathrm{OH}) \mathrm{D}$ measurement. Conclusions. We generated and validated centile curves of $25(\mathrm{OH}) \mathrm{D}$ in the general adult Caucasian population. These curves can help rank vitamin $\mathrm{D}$ centile independently of when $25(\mathrm{OH}) \mathrm{D}$ is measured.

\section{Introduction}

Vitamin D deficiency causes rickets in infants and osteomalacia in adults and contributes to osteoporotic fracture risk [1]. Molecular and epidemiological associations of vitamin D deficiency with other diseases such as cancers, cardiovascular disease, diabetes, depression, and multiple sclerosis have been reported [2-5]. Guessous recently discussed in this journal the relationship of vitamin $\mathrm{D}$ with extraskeletal complications [6].

Serum 25-hydroxyvitamin D $(25(\mathrm{OH}) \mathrm{D})$ is the major circulating form of vitamin D. It is currently the best marker of vitamin $\mathrm{D}$ status and is used by clinicians to determine a patient's vitamin D status [7]. While the definitions of adequate vitamin $\mathrm{D}$ levels differ, several reports suggested that vitamin D levels are low and vitamin D deficiency is 
very prevalent in general adult populations [8]. For example, in Switzerland (latitude $46^{\circ}$ ), the prevalence of vitamin D insufficiency $(25(\mathrm{OH}) \mathrm{D}$ serum levels between $50 \mathrm{nmol} / \mathrm{L}$ and $75 \mathrm{nmol} / \mathrm{L})$ and deficiency $(<50 \mathrm{nmol} / \mathrm{L})$ is $36 \%$ and $38 \%$, respectively [9].

Most of the circulating vitamin $\mathrm{D}$ is synthesized from cholesterol following exposure to sunlight ultraviolet B (UVB), whereas a smaller amount is derived from diet and dietary supplements [2]. Because diet is a naturally low source of vitamin D, vitamin D levels are highly dependent on UVB exposure, even more in settings where food fortification policies are not implemented. Other factors such as body mass index (BMI), skin pigmentation, and geographical factors (e.g., latitude, altitude, and meteorological conditions) can influence circulating vitamin D levels [10].

The physiological mechanisms of seasonal variation in vitamin $\mathrm{D}$ levels are well described $[11,12]$. In studies that collected seasonal data, the highest $25(\mathrm{OH}) \mathrm{D}$ levels were found in summer and autumn and lowest levels in winter and spring $[9,11,13-16]$.

Gender, age, and fat mass seem to influence $25(\mathrm{OH}) \mathrm{D}$ seasonal variations [17]. Surprisingly, international and national guidelines on vitamin $\mathrm{D}$ measure, classification, and substitution do not include seasonal variation in their recommendations [18-20]. A major clinical implication is that seasonal variation of $25(\mathrm{OH}) \mathrm{D}$ can directly influence the diagnosis of vitamin $\mathrm{D}$ insufficiency and deficiency. Currently, a measure of serum $25(\mathrm{OH}) \mathrm{D}$ concentration performed at a given period of the year by a healthcare provider is used to reflect the patient's vitamin D status for the overall year although $25(\mathrm{OH}) \mathrm{D}$ levels could be meaningfully different. Two previous studies that assessed the effects of seasonal variation of $25(\mathrm{OH}) \mathrm{D}$ on the assessment of vitamin $\mathrm{D}$ status in New Zealand confirmed the need of seasonally adjusted thresholds for the diagnosis of vitamin D deficiency [17, 21]. Many people were predicted to have suboptimal $25(\mathrm{OH}) \mathrm{D}$ concentrations for a substantial proportion of the year, despite having apparently adequate concentrations at the time of testing [17]. We are not aware of such population-based analysis using European data. A recent study showed that, even with a comprehensive set of genetic, anthropometric, dietary, and lifestyle correlates, not more than $32.8 \%$ of the variation in $25(\mathrm{OH}) \mathrm{D}$ could be explained in the EPICGermany study and that food intake was only a weak predictor of $25(\mathrm{OH}) \mathrm{D}$ concentrations, while a strong seasonal fluctuation in $25(\mathrm{OH}) \mathrm{D}$ was shown [22].

Recognizing this, we used data from two large population-based Swiss studies to generate and replicate a simple tool that could help healthcare providers in interpreting a patient's vitamin $\mathrm{D}$ status independently of when the patient's $25(\mathrm{OH}) \mathrm{D}$ was measured, while taking into account age, gender, and BMI. The aim of the project was to find a model that predicts the centiles of the $25(\mathrm{OH}) \mathrm{D}$ distribution given age, sex, BMI, and date of the measurement. Such centile curves allow for more precision compared to usual recommendations since they provide adjusted predictions. Moreover, such model provides an insight of $25(\mathrm{OH}) \mathrm{D}$ levels at a different time point.

\section{Materials and Methods}

2.1. Original Dataset, the CoLaus Study. To generate the tool, we used the data from the CoLaus study (http://www.colaus .ch). The primary aim of the CoLaus study was to assess the prevalence of cardiovascular risk factors in the Caucasian population of Lausanne, Switzerland [23] (latitude $46^{\circ}$ ). The sampling procedure of the CoLaus study has been described elsewhere [23]. Briefly, the CoLaus study was populationbased and included participants aged 35 to 75 years. The complete list of the Lausanne inhabitants aged 35-75 years ( $n=56,694$ in 2003) was provided by the population register of the city and served to sample the participants to the study. A simple, nonstratified random sample of $35 \%$ of the overall population was drawn, 8,121 people were eligible for interview, and 6,188 participants were finally included in the cohort (76.2\%). Recruitment began in June 2003 and ended in May 2006. Data were collected by trained field interviewers using standardized questionnaires.

2.2. Replication Dataset, the Bus Santé Study. To replicate the tool, we used the data from the Bus Santé study. The Bus Santé study is an ongoing cross-sectional population-based study conducted in the State of Geneva (latitude $46^{\circ}$ ), Switzerland (http://epidemiologiepopulation.hug-ge.ch). Participants' recruitment has been described in detail previously [24]. Briefly, a representative sample of noninstitutionalized residents aged 20 and more years is selected independently throughout each year by using a standardized procedure using an annual residential list established by local government. The participation rate was $65 \%$. To match with the CoLaus study period, subjects who participated in the Bus Santé study between 2005 and 2008 were included.

2.3. 25-Hydroxyvitamin $D$ and Covariates. In both studies, venous blood samples were drawn after an overnight fast and stored at $-80^{\circ} \mathrm{C}$. In the CoLaus study, serum $25(\mathrm{OH}) \mathrm{D}$ was measured in a single center by an ultra high pressure liquid chromatography-tandem mass spectrometry (LC-MS/MS) system [25]. In the Bus Santé study, serum $25(\mathrm{OH}) \mathrm{D}$ was measured in a single center by using a commercial one-step immunoassay (Architect 25(OH)D, Abbott Architect i2000sr). The correlation between these two methods was determined using 125 serum samples; a Passing-Bablok regression was performed, resulting in an intercept of $8.7195 \%$ CI $[3.31 ; 13.55]$ a slope of 0.86 [0.76; 0.95$]$ (see Figure S1 in Supplementary Material available online at http://dx.doi.org/10.1155/2015/168189), which indicates that LC-MS/MS measurements are slightly lower than Architect ones if values are higher than $62.07 \mathrm{nmol} / \mathrm{L}$. For lower values, Architect measurements are higher.

In both studies, weight and height were measured using standardized procedures. BMI was defined as weight $/$ height ${ }^{2}$ $\left[\mathrm{kg} / \mathrm{m}^{2}\right]$. In the CoLaus study, information on personal medicines, including prescription and self-prescribed drugs, vitamin, mineral supplements, use of oral contraception, and hormonal replacement therapy, was collected. This information was not available in the Bus Santé study. The date of participation was recorded in both studies. 
The CoLaus study and the Bus Santé study complied with the Declaration of Helsinki and were approved by the local Institutional Ethics Committees. All participants gave written informed consent.

2.4. Statistical Analysis. To predict the centiles of the $25(\mathrm{OH}) \mathrm{D}$ distribution, we applied linear regression models on the square-root transformed $25(\mathrm{OH}) \mathrm{D}$ values. Since normality and a constant variance (estimated via the residual variance from the regression) are approximately achieved on the transformed scale, it is possible to estimate any centile of the distribution. A back transformation then returns these centiles on the original scale. We considered various models to adjust for known determinants of $25(\mathrm{OH}) \mathrm{D}$ including gender, age, BMI, and the day when the measurement was made (via sine and cosine), including possible interactions between them, and we selected the best model according to Bayesian information criterion (BIC). In particular, the effect of age was negligible once adjusted for the other characteristics. Thus, age was not included in the final model, whereas some interactions between BMI and sex and between date of measurement and sex were included. Note that the BMI was included as $-1 / \sqrt{ } \mathrm{BMI}$ in our model to limit the influence of individuals with large BMI. This transformation has been used by other authors to transform variables related to obesity towards normality [26]. Because skin pigmentation influences vitamin $\mathrm{D}$ synthesis, analyses were limited to Caucasians. 25(OH)D levels are influenced by vitamin $\mathrm{D}$ therapy and supplementation. We therefore excluded participants from the original dataset who reported vitamin D therapy and supplementation (3.9\% of the original dataset). In both datasets, only participants aged between 35 and 65 years with a BMI between 18 and $40 \mathrm{~kg} / \mathrm{m}^{2}$ were kept, which reduced the sample sizes for the analyses. These BMI limits approximately correspond to percentiles $1 \%$ and 99\%. Like elderly (i.e., >75 years old) participants, subjects with extreme BMI were excluded as they are more likely to suffer from an underlying disease. To account for the difference between the two 25(OH)D measurement methods, we transformed the $25(\mathrm{OH}) \mathrm{D}$ values from the Bus Santé study according to the Passing-Bablok regression analysis (on the square-root transformed $25(\mathrm{OH}) \mathrm{D}$ values) performed on 125 random serums analyzed with the two methods.

The best model based on BIC was given as follows:

$$
\begin{aligned}
& \sqrt{25(\mathrm{OH}) \mathrm{D}}=\beta_{0}+\beta_{1} \sin \left(\frac{2 \pi(\text { day }-1)}{365}\right)+\beta_{2} \\
& \cdot \cos \left(\frac{2 \pi(\text { day }-1)}{365}\right) \\
& +\beta_{3} \sin \left(\frac{4 \pi(\text { day }-1)}{365}\right)+\beta_{4} \cos \left(\frac{4 \pi(\text { day }-1)}{365}\right) \\
& +\beta_{5} t(\mathrm{BMI})+\beta_{6} t(\mathrm{BMI})^{2} \\
& +\beta_{7} I(\text { male }) I(\mathrm{BMI}<25)(t(\mathrm{BMI})-t(25))^{2}
\end{aligned}
$$

$$
\begin{aligned}
& \left.+\beta_{8} I \text { (female }\right) \sin \left(\frac{2 \pi(\text { day }-1)}{365}\right) \\
& \left.+\beta_{9} I \text { (female }\right) \cos \left(\frac{2 \pi(\text { day }-1)}{365}\right) .
\end{aligned}
$$

BMI was transformed using an inverse square-root function $t(x)=-1 / \sqrt{ } x$.

Model's coefficients were as follows: $\beta_{0}=-2.754$ ( $P$ value $=0.295), \beta_{1}=-1.077(P$ value $<0.0001), \beta_{2}=-0.756(P$ value $<0.0001), \beta_{3}=0.188(P$ value $<0.0001), \beta_{4}=0.025(P$ value $=0.368), \beta_{5}=-81.08(P$ value $=0.002), \beta_{6}=-165.6(P$ value $=0.014), \beta_{7}=-1174(P$ value $\leq 0.0001), \beta_{8}=0.218(P$ value $<0.0001)$, and $\beta_{9}=0.164(P$ value $=0.003)$. The model explained $29 \%$ of the variance.

Of note, the equation $\beta_{1} \sin (2 \pi($ day -1$) / 365)+$ $\beta_{2} \cos (2 \pi($ day -1$) / 365)$ is equivalent to $\beta_{1}^{\prime} \sin (2 \pi$ (day $\left.1) / 365+\beta_{2}^{\prime}\right)$ that has been used in previous work by Bolland et al. [21] with $\beta_{1}=\beta_{1}^{\prime} \cos \left(\beta_{2}^{\prime}\right)$ and $\beta_{2}=\beta_{1}^{\prime} \sin \left(\beta_{2}^{\prime}\right)$. In our model, we added two supplementary sine-cosine terms that allow the time between minimum and maximum levels to be different from 6 months. The lowest predicted values were at mid-March and the highest at mid-August.

We then used the standard deviation (SD) of the residuals $\left(\sigma_{\epsilon}=1.36\right)$ to predict all centiles of the $25(\mathrm{OH}) \mathrm{D}$ distribution given fixed values for sex, BMI, and day of measurement. The $\alpha$ centile is given as $\widehat{y}+z_{\alpha} \sigma_{\epsilon}$, where $\widehat{y}$ is the fitted value and $z_{\alpha}$ is the $\alpha$ quantile of a standard normal distribution. The square of this centile returns the value on the original scale.

To validate the model, we accounted for the fact that some participants in the Bus Santé had vitamin D supplementation (without knowing which ones). We refitted our regression model above on the CoLaus participants with and without vitamin $\mathrm{D}$ supplementation $(N=5,066)$ and used these model coefficients to estimate the $25(\mathrm{OH}) \mathrm{D}$ values in the Bus Santé study. For each observation, we estimated the corresponding quantile. Histogram and empirical cumulative distribution function of the quantiles of the Bus Santé observations predicted with the model based on the CoLaus data were inspected. Quantiles should be approximatively uniformly distributed between 0 and 1 if the model is correct.

\section{Results}

Overall, 4,912 subjects were included in the original dataset (CoLaus study). Participants' characteristics are shown in Table 1. The mean (SD) age and mean BMI were 49.8 (8.5) years and $25.6(4.1) \mathrm{kg} / \mathrm{m}^{2}$, respectively. Fifty \% (2491/4912) of the participants were women, $14.3 \%$ (703/4912) were obese (i.e., BMI $\geq 30)$, and 10.1\% (251/2491) of women used oral contraceptive or hormonal replacement. The number (\%) of participants by month varied from 268 (5.5\%) to $526(10.7 \%)$ (see Table S1) with fewer people measured during the summer months (May to August).

The mean (SD) 25(OH)D level was 47.6 (22.1) nmol/L (Table 1$)$. The mean $25(\mathrm{OH}) \mathrm{D}$ levels differed by season, gender, and BMI (see Table S2). Overall, 25(OH)D levels 


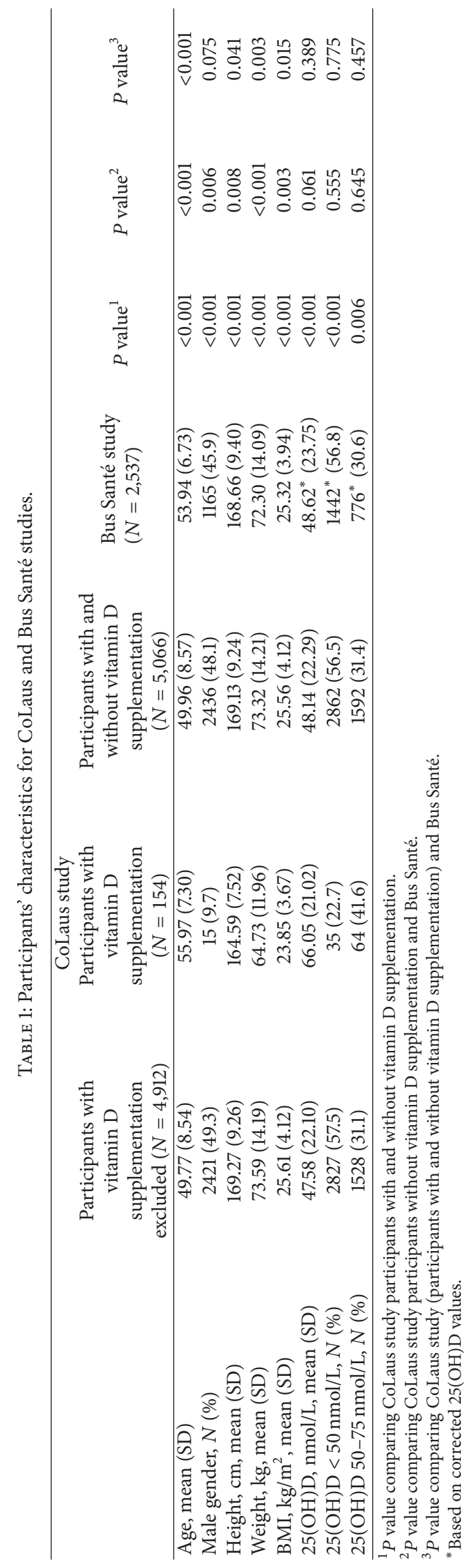


were $<50 \mathrm{nmol} / \mathrm{L}$ and $<75 \mathrm{nmol} / \mathrm{L}$ in $57.5 \%$ and $31.1 \%$ of the participants.

Centile curves for $25(\mathrm{OH}) \mathrm{D}$ predicted with the multivariate model as a function of date of measurement for men and women with a fixed value of BMI are illustrated in Figure 1 expressed as a function of month of measurements and in Figure S2 expressed as a function of week of measurements. The interaction between gender and BMI appears clearly when looking at the quantiles predicted as a function of the BMI for a given day of measurement, as presented in Figure S3. August 22 is found to be the day with the highest predicted $25(\mathrm{OH}) \mathrm{D}$ values, for both men and women. The lowest predicted levels are on March 11 for men and on March 18 for women. This difference is due to the sex-day interaction.

Given values for sex, BMI, and day of measurement, it is possible to estimate any centile of the $25(\mathrm{OH}) \mathrm{D}$ distribution. For example, the median $25(\mathrm{OH}) \mathrm{D}$ value measured on August 22 of a woman with a BMI of 20 is $69.1 \mathrm{nmol} / \mathrm{L}$. Another important aspect of this model is that it can be used to estimate the quantile corresponding to a $25(\mathrm{OH}) \mathrm{D}$ measurement. For example, a man with a BMI of 25 and a $25(\mathrm{OH}) \mathrm{D}$ level of $60 \mathrm{nmol} / \mathrm{L}$ measured on January 1 will be on the $88.6 \%$ quantile.

Our model fitted the data pretty well, as can be seen in Figure S4, where the histogram and the empirical cumulative distribution function of the quantiles predicted by the model were almost those of a uniform distribution.

3.1. Replication. Overall, 2,537 subjects were included in the replication dataset (Bus Santé study). Participants' characteristics are shown in Table 1 . The replication sample differed from the original in several ways. The number (\%) of participants by month varied from 59 (2.3\%) to 298 (11.8\%) (see Table S1).

The uncorrected mean $25(\mathrm{OH}) \mathrm{D}$ values were higher in the Bus Santé than in CoLaus. The mean (SD) 25(OH)D level was $50.5(19.9) \mathrm{nmol} / \mathrm{L}$. The mean 25(OH)D levels differed by season, gender, and BMI (see Table S2). After correction for difference in methods used, the mean (SD) 25(OH)D value in the Bus Santé was similar to the mean 25(OH)D in CoLaus ( $48.6 \mathrm{nmol} / \mathrm{L}$ versus $48.1 \mathrm{nmol} / \mathrm{L}, P$ value $=0.39$, see Table 1$)$. Histogram and empirical cumulative distribution function of the quantiles of the Bus Santé observations predicted with the model based on the CoLaus were very close to uniformity suggesting that the model is correct (see Figure S5). To check the validity of our prediction, the predicted percentage of people under a given quantile can be considered. For example, $12.1 \%$ of participants have a predicted $25(\mathrm{OH}) \mathrm{D}$ value under the $10 \%$ centile, or $4.1 \%$ are predicted under the $3 \%$ centile. These proportions are slightly overestimated (as shown in the histogram in Figure S5) but close to correct though. The highest difference between measured and predicted quantiles is given by the Kolmogorov-Smirnov test statistic, which was equal to 0.036 (95\% CI [0.026; 0.054]).

\section{Discussion}

Using two large Swiss population-based studies, we generated and validated centile curves of $25(\mathrm{OH}) \mathrm{D}$ in the general adult population. These curves can help healthcare providers in interpreting a patient's vitamin $\mathrm{D}$ status independently of when the patient's $25(\mathrm{OH}) \mathrm{D}$ was measured. We provided gender- and BMI-specific $25(\mathrm{OH}) \mathrm{D}$ centile curves as $25(\mathrm{OH}) \mathrm{D}$ level is influenced by these factors. We developed a free online tool available at http://www.iumsp.ch/charts/ select_graph.htm that can be used to determine where a patient fits compared to a reference population at a given time.

Given the growing evidence of associations of low vitamin D levels with several chronic diseases, determining vitamin D status has gained increasing importance [27], especially in settings such as in Switzerland where no vitamin D fortification policy is implemented. Efforts to find a sufficiently predictive model for vitamin $\mathrm{D}$ status without using information on $25(\mathrm{OH}) \mathrm{D}$ level have failed and currently there is no substitute for $25(\mathrm{OH}) \mathrm{D}$ testing [28]. While the best determinant of vitamin $\mathrm{D}$ status is the serum concentration of $25(\mathrm{OH}) \mathrm{D}$, this parameter fluctuates with season and not considering season-specific $25(\mathrm{OH}) \mathrm{D}$ levels can mislead the diagnosis of vitamin D status. Two studies conducted in New Zealand previously assessed the effects of seasonal variation of $25(\mathrm{OH}) \mathrm{D}$ on diagnosis of vitamin $\mathrm{D}$ sufficiency $[17,21]$. In the most recent one, Bolland et al. used the data from postmenopausal women $(N=1,606)$ and from a small sample $(N=378)$ of middle-aged and older men and found that many people were predicted to have suboptimal $25(\mathrm{OH}) \mathrm{D}$ concentrations for a substantial proportion of the year, despite having apparently adequate concentrations at the time of testing [21]. Results observed in New Zealand are however not directly transposable to European settings given that $25(\mathrm{OH}) \mathrm{D}$ levels are influenced by latitude and sunshine duration. Brown et al. proposed an interesting mathematical model of $25(\mathrm{OH}) \mathrm{D}$ serum using information on food, supplements, sun, and 25(OH)D levels [29]. Yet, information on $25(\mathrm{OH}) \mathrm{D}$ levels dated from 1998, and compared to this present study, sample size was smaller and BMI was not taken into account. Thus, our work provides information using more recent data collected in a larger study sample from Switzerland, a country situated in the center of Europe.

Furthermore, the number of large population-based studies reporting seasonal data is limited $[8,15,16,30]$, and we found no study that reported centile curves. Centile curves present several advantages. Centile curves are routinely used in clinical medicine to detect extreme values of a measurement of interest $[31,32]$. Data used to construct the curves are representative of the reference population. Centile curves can take into consideration major factors (e.g., gender, BMI, and date of measurement) that influence the distribution. In line with a previous Swiss population-based study [9], age was not independently associated with $25(\mathrm{OH}) \mathrm{D}$. The lack of an independent association might be explained, at least in part, by the upper age limit of the participants (i.e., 75 years) included in the CoLaus and Bus Santé studies and/or by the adjustment for BMI. Age-related vitamin D deficiency has been essentially reported among elderly subjects; cutaneous production of vitamin D is decreased [33] and exposure to sunshine through outdoor activities reduced in elderly subjects [34]. The effect of age on vitamin D deficiency among 

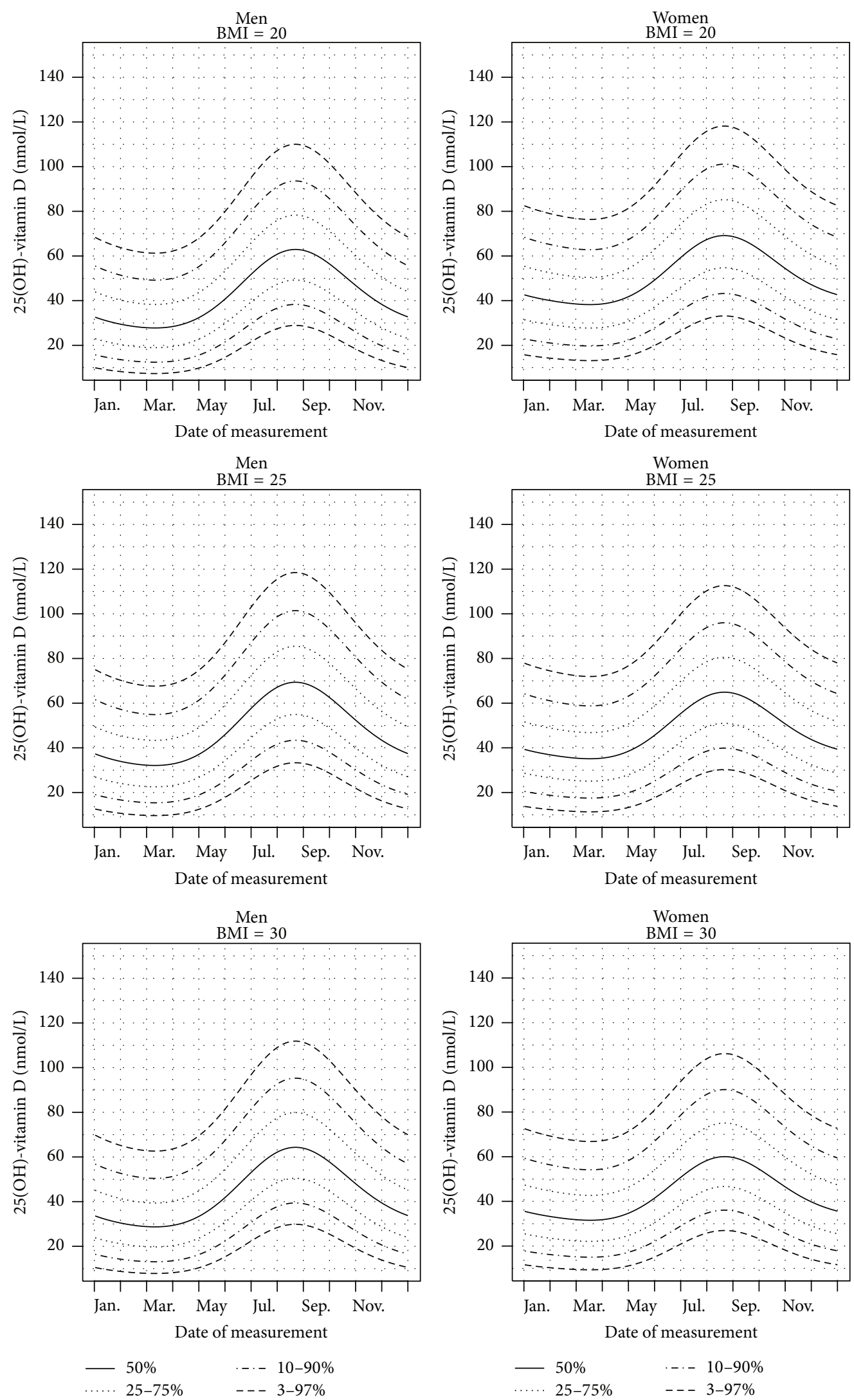

Figure 1: Quantiles of 25(OH)D according to the date of measurement (horizontal units expressed in month of measurements), for men and women with different BMI values (20, 25, and 30). 
subjects younger than 75 years might be not strong enough to be captured after adjustment for gender, BMI, and day of measurement. BMI frequently increases with age [35] and there are several mechanisms by which vitamin $\mathrm{D}$ levels could be inversely associated with BMI including the decrease of outdoor physical activity, the inadequate diet, and the sequestration of vitamin $\mathrm{D}$ in the cutaneous fat among people with overweight or obesity [36]. Our results suggest that BMI and not age is worth considering when interpreting seasonal variation of $25(\mathrm{OH}) \mathrm{D}$. Nevertheless, only adult participants between 35 and 65 years were included. When considering children, adolescents, and elderly people, the age association might be different from what was observed in the present study.

While not considering season-specific 25(OH)D levels biases the assessment of vitamin $\mathrm{D}$ status, the benefit of maintaining optimal $25(\mathrm{OH}) \mathrm{D}$ levels throughout the seasons remained to be demonstrated. There is some evidence suggesting that cycles of suboptimal level of vitamin D throughout the year are harmful for bone health [37-39], but such evidence for other health outcomes is little [11, 40]. The observation that not only the parathyroid hormone but also the active form of vitamin $\mathrm{D}$ (i.e., 1,25(OH)2D) parallels $25(\mathrm{OH}) \mathrm{D}$ seasonal variation [41] suggests that variation, if meaningful enough, can influence health outcomes. In general, any period of vitamin $\mathrm{D}$ insufficiency is undesirable $[17,21,42]$.

Finally, given the cost of $25(\mathrm{OH}) \mathrm{D}$ testing and the evidence of overtesting, some insurance and regions have altered their policies to restrict $25(\mathrm{OH}) \mathrm{D}$ testing $[28,43]$. In the absence of vitamin $\mathrm{D}$ supplementation, our simple tool, which relies on a single $25(\mathrm{OH}) \mathrm{D}$ test, could potentially decrease healthcare cost by limiting the number of $25(\mathrm{OH}) \mathrm{D}$ tests performed for a given patient throughout a year to determine vitamin $\mathrm{D}$ status.

4.1. Strengths and Limitations. When interpreting the findings of this study, one has to keep in mind its limitations. First, our results are representative of general and unselected adult populations in Switzerland. Because vitamin D levels have been shown to vary between populations, the centile curves presented in this report are not generalizable to populations that differ from our source population in terms of both individual- (e.g., age, ethnicity) and contextualdeterminants of vitamin D levels (e.g., food fortification policy). Of note, these results are latitude-specific as both studies were conducted at the same latitude. When determinants of vitamin D levels are similar, our results can potentially be extrapolated to European countries within the same range of latitude $\left(45^{\circ}-50^{\circ}\right)$ such as France, Austria, the southern part of Germany, the northern part of Italy, and some eastern European countries. For other countries, our method could be replicated when population-based data are available. Second, we relied on a single measure of vitamin $\mathrm{D}$ per participant. Third, as previous studies [17, 21], if we want to predict future values of an individual along the year given a measurement made on a given day, we have to assume that each participant's $25(\mathrm{OH}) \mathrm{D}$ concentrations throughout the year would follow a curve similar to that of the overall population; that is, the correlation between two repeated measurements made on the same subjects in different seasons would be perfect (1.0). To be able to affine such individual predictions taking into account that this correlation is not perfect, data from a longitudinal analysis would be necessary. While our model was built to fit the seasonal variation of $25(\mathrm{OH}) \mathrm{D}$ at the population level, seasonal variation at the individual level can differ, notably in individuals who differ meaningfully from the study population in terms of vitamin $\mathrm{D}$ levels determinants. Fourth, we lack information on vitamin $\mathrm{D}$ therapy or supplementation in the replication dataset. We corrected the replication dataset $25(\mathrm{OH}) \mathrm{D}$ levels and centile curves using original dataset derived correction factor. Moreover, the measurement methods were different between the two datasets.

Another limitation could be the possible time trends of $25(\mathrm{OH}) \mathrm{D}$ levels from the data collection up to now. Samples were collected almost 10 years ago and we cannot exclude that population-averaged levels have changed. Yet, trends analyses of $25(\mathrm{OH}) \mathrm{D}$ levels in the Swiss population suggest that levels have been stable [9].

Our study has several strengths. To generate centile curves, we used standard reference 25(OH)D method of measurement (LC-MS/MS). We replicated our findings using a similar yet different population and a standard commercial immunoassay. The city of Lausanne (CoLaus study) and the state of Geneva (Bus Santé study) are located 37 miles away on the same latitude. Reference populations are ideally composed of individuals assumed to be "normal." We used data from noninstitutionalized residents randomly selected to participate in population-based studies as reference populations. Compared to other study designs (clinical trials, hospital data, and institutions data), this approach mitigates the risk of having unrepresentative populations. Finally, we did not rely on healthcare-related administrative data, which are subject to bias including seasonal testing bias [28].

In conclusion, we used a centile curves approach, which is well known with growth charts, to generate and validate a model available as a free online tool that could help in interpreting vitamin $\mathrm{D}$ status independently of when $25(\mathrm{OH}) \mathrm{D}$ is measured.

\section{Conflict of Interests}

The authors declare that there is no conflict of interests regarding the publication of this paper.

\section{References}

[1] M. F. Holick, "Vitamin D: important for prevention of osteoporosis, cardiovascular heart disease, type 1 diabetes, autoimmune diseases, and some cancers," Southern Medical Journal, vol. 98, no. 10, pp. 1024-1027, 2005.

[2] M. F. Holick, "Vitamin D deficiency," The New England Journal of Medicine, vol. 357, no. 3, pp. 266-281, 2007.

[3] I. Guessous, M. Bochud, O. Bonny, and M. Burnier, "Calcium, vitamin D and cardiovascular disease," Kidney and Blood Pressure Research, vol. 34, no. 6, pp. 404-417, 2011. 
[4] S. Penckofer, J. Kouba, D. E. Wallis, and M. A. Emanuele, "Vitamin D and diabetes: let the sunshine in," Diabetes Educator, vol. 34, no. 6, pp. 939-954, 2008.

[5] E. R. Bertone-Johnson, "Vitamin D and the occurrence of depression: causal association or circumstantial evidence?" Nutrition Reviews, vol. 67, no. 8, pp. 481-492, 2009.

[6] I. Guessous, "Role of vitamin D deficiency in extraskeletal complications: predictor of health outcome or marker of health status?" BioMed Research International, vol. 2015, Article ID 563403, 13 pages, 2015.

[7] K. A. Kennel, M. T. Drake, and D. L. Hurley, "Vitamin D deficiency in adults: when to test and how to treat," Mayo Clinic Proceedings, vol. 85, no. 8, pp. 752-758, 2010.

[8] N. M. van Schoor and P. Lips, "Worldwide vitamin D status," Best Practice and Research: Clinical Endocrinology and Metabolism, vol. 25, no. 4, pp. 671-680, 2011.

[9] I. Guessous, V. Dudler, N. Glatz et al., "Vitamin D levels and associated factors: a population-based study in Switzerland," Swiss Medical Weekly, vol. 142, 2012.

[10] M. F. Holick, "Vitamin D: a millenium perspective," Journal of Cellular Biochemistry, vol. 88, no. 2, pp. 296-307, 2003.

[11] J. D. Maxwell, "Seasonal variation in vitamin D," Proceedings of the Nutrition Society, vol. 53, no. 3, pp. 533-543, 1994.

[12] A. R. Webb, L. Kline, and M. F. Holick, "Influence of season and latitude on the cutaneous synthesis of vitamin D3: exposure to winter sunlight in Boston and Edmonton will not promote vitamin D3 synthesis in human skin," Journal of Clinical Endocrinology and Metabolism, vol. 67, no. 2, pp. 373-378, 1988.

[13] J. Klenk, K. Rapp, M. D. Denkinger et al., "Seasonality of vitamin D status in older people in Southern Germany: implications for assessment," Age and Ageing, vol. 42, no. 3, Article ID aft042, pp. 404-408, 2013.

[14] M. Kull Jr., R. Kallikorm, A. Tamm, and M. Lember, "Seasonal variance of $25-(\mathrm{OH})$ vitamin $\mathrm{D}$ in the general population of Estonia, a Northern European country," BMC Public Health, vol. 9, article 22, 2009.

[15] Z. Lagunova, L. C. Porojnicu, F. Lindberg, S. Hexeberg, and J. Moan, "The dependency of vitamin D status on body mass index, gender, age and season," Anticancer Research, vol. 29, no. 9, pp. 3713-3720, 2009.

[16] S. Levis, A. Gomez, C. Jimenez et al., "Vitamin D deficiency and seasonal variation in an adult south Florida population," Journal of Clinical Endocrinology and Metabolism, vol. 90, no. 3, pp. 1557-1562, 2005.

[17] M. J. Bolland, A. B. Grey, R. W. Ames et al., "The effects of seasonal variation of 25 -hydroxyvitamin $\mathrm{D}$ and fat mass on a diagnosis of vitamin D sufficiency," The American Journal of Clinical Nutrition, vol. 86, no. 4, pp. 959-964, 2007.

[18] WHO, Vitamin and Mineral Requirements in Human Nutrition, World Health Organization, Geneva, Switzerland, 2nd edition, 2005, http://www.who.int/nutrition/publications/mi-cro-nutrients/9241546123/en/.

[19] Federal Office for Public Health and Federal Commission for Nutrition, Vitamin D Deficiency: Evidence, Safety, and Recommendations for the Swiss Population. Expert Report of the FCN, Federal Office for Public Health, Zürich, Switzerland, 2012, http://www.bag.admin.ch/org/.

[20] IOM, Dietary Reference Intakes for Calcium and Vitamin D, 2010, http://www.iom.edu/Reports/2010/Dietary-ReferenceIntakes-for-calcium-and-vitamin-D.aspx.
[21] M. J. Bolland, W. W. Chiu, J. S. Davidson et al., "The effects of seasonal variation of 25-hydroxyvitamin $\mathrm{D}$ on diagnosis of vitamin D insufficiency," New Zealand Medical Journal, vol. 121, no. 1286, pp. 63-74, 2008.

[22] T. Kühn, R. Kaaks, B. Teucher et al., "Dietary, lifestyle, and genetic determinants of vitamin D status: a cross-sectional analysis from the European Prospective Investigation into Cancer and Nutrition (EPIC)-Germany study," European Journal of Nutrition, vol. 53, no. 3, pp. 731-741, 2014.

[23] M. Firmann, V. Mayor, P. M. Vidal et al., "The CoLaus study: a population-based study to investigate the epidemiology and genetic determinants of cardiovascular risk factors and metabolic syndrome," BMC Cardiovascular Disorders, vol. 8, article 6, 2008.

[24] I. Guessous, M. Bochud, J.-M. Theler, J.-M. Gaspoz, and A. Pechère-Bertschi, "1999-2009 Trends in prevalence, unawareness, treatment and control of hypertension in Geneva, Switzerland," PLoS ONE, vol. 7, no. 6, Article ID e39877, 2012.

[25] S. J. Bruce, B. Rochat, A. Béguin et al., "Analysis and quantification of vitamin D metabolites in serum by ultra-performance liquid chromatography coupled to tandem mass spectrometry and high-resolution mass spectrometry-a method comparison and validation," Rapid Communications in Mass Spectrometry, vol. 27, no. 1, pp. 200-206, 2013.

[26] T. Gasser, P. Ziegler, B. Seifert, A. Prader, L. Molinari, and R. Largo, "Measures of body mass and of obesity from infancy to adulthood and their appropriate transformation," Annals of Human Biology, vol. 21, no. 2, pp. 111-125, 1994.

[27] A. M. Rose, "Vitamin D testing: clinical and laboratory considerations," Medical Laboratory Observer, vol. 45, no. 5, pp. 8-16, 2013.

[28] A. N. Peiris, B. A. Bailey, B. N. Guha, R. Copeland, and T. Manning, "Can a model predictive of vitamin D status be developed from common laboratory tests and demographic parameters?" Southern Medical Journal, vol. 104, no. 9, pp. 636639, 2011.

[29] J. Brown, A. Ignatius, M. Amling, and F. Barvencik, "New perspectives on vitamin D sources in Germany based on a novel mathematical bottom-up model of 25(OH)D serum concentrations," European Journal of Nutrition, vol. 52, no. 7, pp. 1733-1742, 2013.

[30] T. Hagenau, R. Vest, T. N. Gissel et al., "Global vitamin D levels in relation to age, gender, skin pigmentation and latitude: an ecologic meta-regression analysis," Osteoporosis International, vol. 20, no. 1, pp. 133-140, 2009.

[31] E. M. Wright and P. Royston, "Calculating reference intervals for laboratory measurements," Statistical Methods in Medical Research, vol. 8, no. 2, pp. 93-112, 1999.

[32] E. M. Wright and P. Royston, "A comparison of statistical methods for age-related reference intervals," Journal of the Royal Statistical Society Series A: Statistics in Society, vol. 160, no. 1, pp. 47-69, 1997.

[33] M. F. Holick, L. Matsuoka, and J. Wortsman, "Age, vitamin D, and solar ultraviolet," The Lancet, vol. 334, no. 8671, pp. 1104$1105,1989$.

[34] F. M. Gloth III, C. M. Gundberg, B. W. Hollis, J. G. Haddad Jr., and J. D. Tobin, "Vitamin D deficiency in homebound elderly persons," Journal of the American Medical Association, vol. 274, no. 21, pp. 1683-1686, 1995.

[35] Z. Welon, A. Szklarska, T. Bielicki, and R. M. Malina, "Sex differences in the pattern of age-dependent increase in the BMI 
from 20-59 years," American Journal of Human Biology, vol. 14, no. 6, pp. 693-698, 2002.

[36] J. Wortsman, L. Y. Matsuoka, T. C. Chen, Z. Lu, and M. F. Holick, "Decreased bioavailability of vitamin D in obesity," The American Journal of Clinical Nutrition, vol. 72, no. 3, pp. 690693, 2000.

[37] P. B. Rapuri, H. K. Kinyamu, J. C. Gallagher, and V. Haynatzka, "Seasonal changes in calciotropic hormones, bone markers, and bone mineral density in elderly women," The Journal of Clinical Endocrinology \& Metabolism, vol. 87, no. 5, pp. 2024-2032, 2002.

[38] H. T. Viljakainen, A. Palssa, M. Kärkkäinen et al., "A seasonal variation of calcitropic hormones, bone turnover and bone mineral density in early and mid-puberty girls-a cross-sectional study," British Journal of Nutrition, vol. 96, no. 1, pp. 124-130, 2006.

[39] T. R. Hill, D. McCarthy, J. Jakobsen, C. Lamberg-Allardt, M. Kiely, and K. D. Cashman, "Seasonal changes in vitamin D status and bone turnover in healthy Irish postmenopausal women," International Journal for Vitamin and Nutrition Research, vol. 77, no. 5, pp. 320-325, 2007.

[40] A. Porojnicu, T. E. Robsahm, J. P. Berg, and J. Moan, "Season of diagnosis is a predictor of cancer survival. Sun-induced vitamin D may be involved: a possible role of sun-induced Vitamin D," The Journal of Steroid Biochemistry and Molecular Biology, vol. 103, no. 3-5, pp. 675-678, 2007.

[41] S. S. Sherman, B. W. Hollis, and J. D. Tobin, "Vitamin D status and related parameters in a healthy population: the effects of age, sex, and season," Journal of Clinical Endocrinology and Metabolism, vol. 71, no. 2, pp. 405-413, 1990.

[42] R. Vieth, "How to optimize vitamin D supplementation to prevent cancer, based on cellular adaptation and hydroxylase enzymology," Anticancer Research, vol. 29, no. 9, pp. 3675-3684, 2009.

[43] K. Bilinski and S. Boyages, "Evidence of overtesting for vitamin $\mathrm{D}$ in Australia: an analysis of 4.5 years of Medicare Benefits Schedule (MBS) data," BMJ Open, vol. 3, no. 6, Article ID 002955, 2013. 


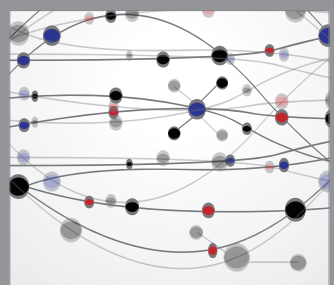

The Scientific World Journal
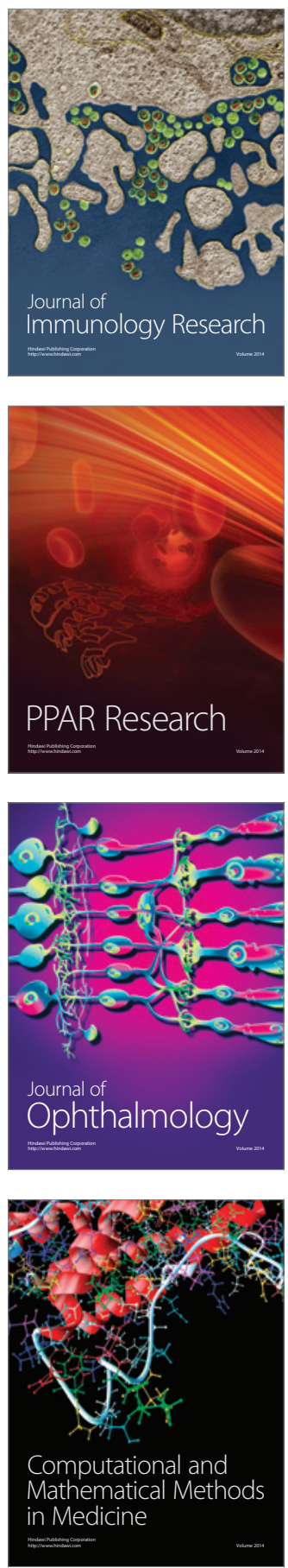

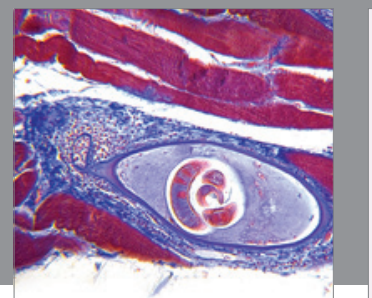

Gastroenterology

Research and Practice
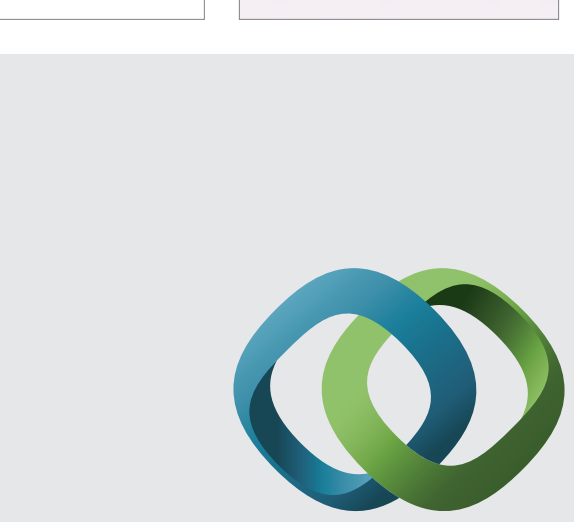

\section{Hindawi}

Submit your manuscripts at

http://www.hindawi.com
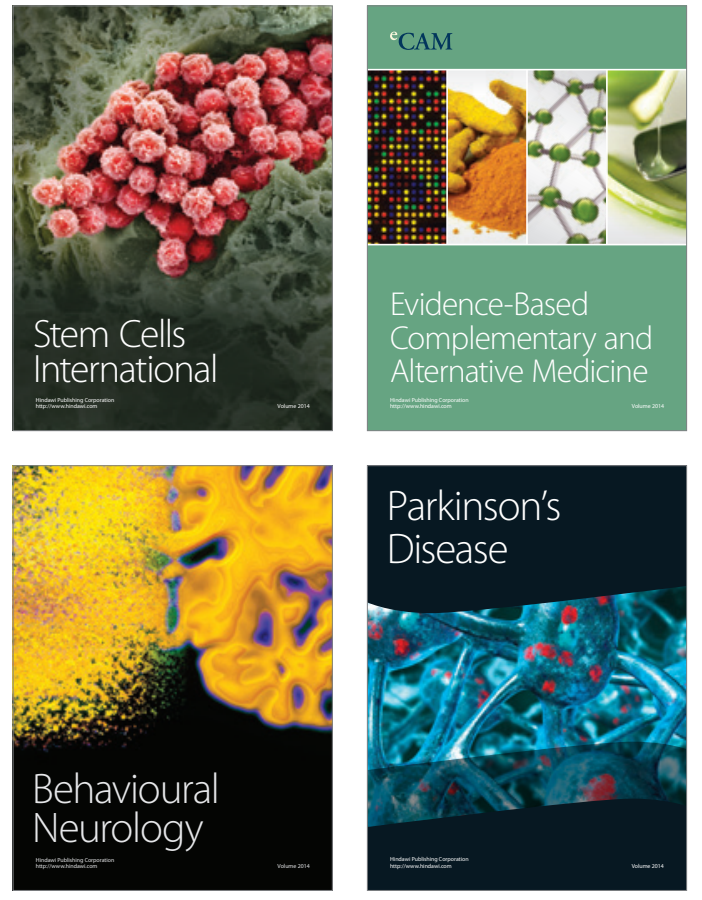
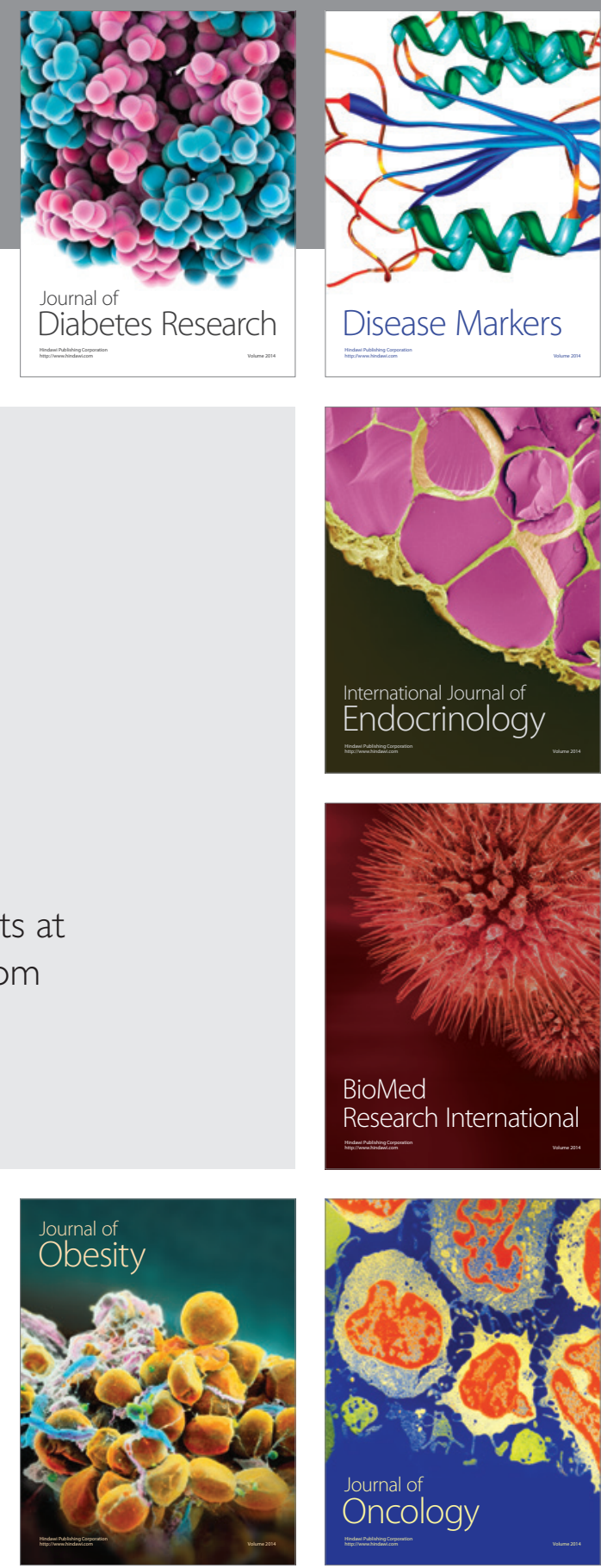

Disease Markers
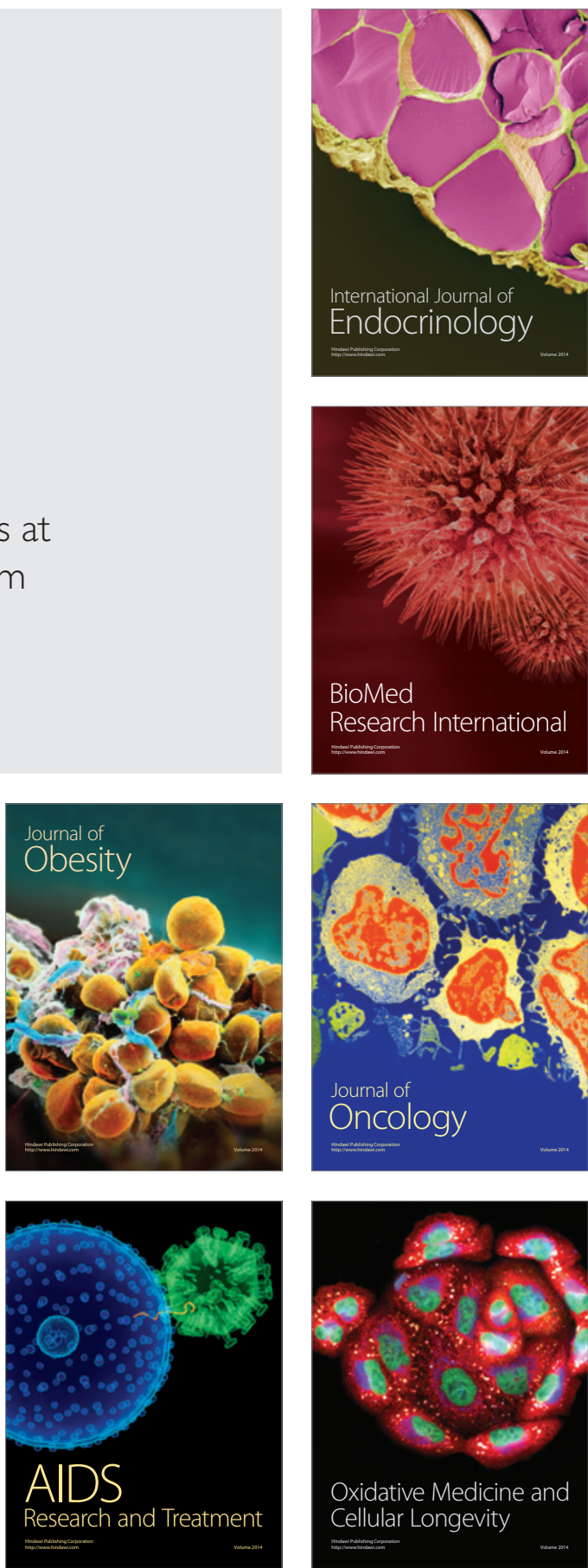